\title{
Purtscher's retinopathy followed by neovascular glaucoma
}

This article was published in the following Dove Press journal:

Clinical Ophthalmology

13 November 2013

Number of times this article has been viewed

\section{Masasko Kuroda' \\ Akihiro Nishida' \\ Masashi Kikuchi \\ Yasuo Kurimoto' \\ 'Department of Ophthalmology, Kobe City Medical Center General \\ Hospital, Kobe, Hyogo, Japan; ${ }^{2}$ Kikuchi \\ Eye Clinic, Kobe, Hyogo, Japan}

Correspondence: Akihiro Nishida

Department of Ophthalmology,

Kobe City Medical Center

General Hospital, 2-I-I, Minatojima-

Minamimachi, Chuo-ku, Kobe City,

Hyogo, 650-0047, Japan

$\mathrm{Tel}+8 \mid 78302432$ I

Fax +8I 783027537

Email aki_n@kcho.jp
Abstract: We report the case of a 66-year-old Japanese man who developed neovascular glaucoma secondary to Purtscher's retinopathy following a head injury. The patient presented at our hospital with blurred vision and a visual field abnormality in his left eye 1 month after suffering from a head injury. Upon initial presentation, his best-corrected visual acuity on a decimal chart was 1.5 oculus dexter and 0.6 oculus sinister. The intraocular pressure (IOP) was $12 \mathrm{mmHg}$ in both eyes. Fundus examination of the left eye revealed multiple white lesions in the posterior pole. Optical coherence tomography demonstrated retinal edema, particularly in the inner retina. On the basis of these findings, a diagnosis of Purtscher's retinopathy was made. One month after the initial examination, the visual acuity in the left eye deteriorated to 0.01 in decimal chart, and the IOP increased to $37 \mathrm{mmHg}$. Gonioscopy showed angle neovascularization. The patient received an intravitreal bevacizumab injection and panretinal photocoagulation. Subsequently, the IOP normalized and the angle neovascularization regressed.

Keywords: blurred vision, visual field, retinal edema, head injury, head trauma

\section{Introduction}

Purtscher's retinopathy is a rare condition observed in patients with a history of trauma without direct globe injury or other systemic diseases. ${ }^{1}$ It was first described in 1910 by Othmar Purtscher, an Austrian ophthalmologist, as angiopathia retinae traumatica. ${ }^{1}$ In his original report, Purtscher theorized that the retinal changes were due to extravasation of the lymph from retinal vessels following increased cerebrospinal pressure during head injury. Today, little more is known, although etiologically, chest compression, childbirth, long bone fracture, orthopedic surgery, acute pancreatitis, renal failure, connective tissue disorders, and many other factors are recognized. ${ }^{2,3}$ Furthermore, it is known that several factors contribute to the pathogenesis of this retinopathy, ${ }^{2,3}$ including blood vessel damage following acute expansion of retinal veins, air and fat embolism, and aggregation of leukocytes, platelets, and fibrin in precapillary arterioles. Acute fundus abnormalities in Purtscher's retinopathy include retinal hemorrhage, optic disc swelling, cotton-wool patch, and multiple retinal whitening between the retinal arterioles and the venules in the inner retina, which are referred to as Purtscher flecken. According to previous reports, visible retinal emboli are absent, and retinal whitening spares the area immediately adjacent to the retinal arterioles., ${ }^{2,3}$ Furthermore, the prognosis is good, with a gradual improvement in visual function that returns to pretraumatic levels within 1-3 months without treatment in most patients., ${ }^{2,3}$ Although retinal neovascularization had been reported to occur following Purtscher's retinopathy, ${ }^{4}$ angle neovascularization has never been reported. Here, we report the case 
of a 66-year-old Japanese man who developed Purtscher's retinopathy followed by neovascular glaucoma after suffering from a head injury.

\section{Case report}

A 66-year-old Japanese man presented to our hospital with blurred vision and a central scotoma in his left eye 1 month after suffering from a head injury, which was reported to have occurred following a blow received on his left forehead during a fight. The patient initially noticed the ophthalmic symptoms 1 week after the injury. According to his medical records after the injury occurred, there were no contusions or subcutaneous hemorrhages in the facial region. Computed tomography of his head revealed no fracture, brain damage, or intracranial hemorrhage. Furthermore, there was no history of bone fracture, strong compressions to the chest and abdomen, or direct globe contusion. Although he had a history of hypertension and angina pectoris that were satisfactorily controlled by beta-blocker therapy, there was no history of diabetes and hyperlipidemia. He was a chronic smoker (20 cigarettes per day for 40 years).

At initial presentation, his best-corrected visual acuity (BCVA) on a decimal chart was 1.5 oculus dexter and 0.6 oculus sinister. The intraocular pressure (IOP) was $12 \mathrm{mmHg}$ in both eyes. The critical flicker frequency was $38 \mathrm{~Hz}$ oculus dexter and $24 \mathrm{~Hz}$ oculus sinister. A relative afferent pupillary defect was present in his left eye. Fundus examination revealed no distinct abnormalities in the right eye; however, it revealed multiple white lesions surrounding the optic disk of the left eye, without retinal neovascularization (Figure 1A). Goldmann perimetry demonstrated paracentral scotomas in the left eye (Figure 1B). Optical coherence tomography demonstrated retinal edema, particularly in the inner retina of the left eye (Figure 1C and D). Furthermore, carotid artery ultrasonography was performed to rule out ocular ischemic syndrome. Although there were slight plaques bilaterally, no significant coarctation was observed. On the basis of these findings, a clinical diagnosis of Purtscher's retinopathy of the left eye was made. The patient was followed up without treatment.

One month after the initial examination, visual acuity in the left eye deteriorated, and the BCVA and IOP became 0.01 in decimal chart and $37 \mathrm{mmHg}$, respectively. Gonioscopy revealed angle neovascularization in all quadrants. The angle was wide open with no peripheral anterior synechiae. In addition, fluorescein angiography demonstrated a delay of 33 seconds in the arm-to-retina circulation time, with a mild leak observed from the peripheral vessels as well as from

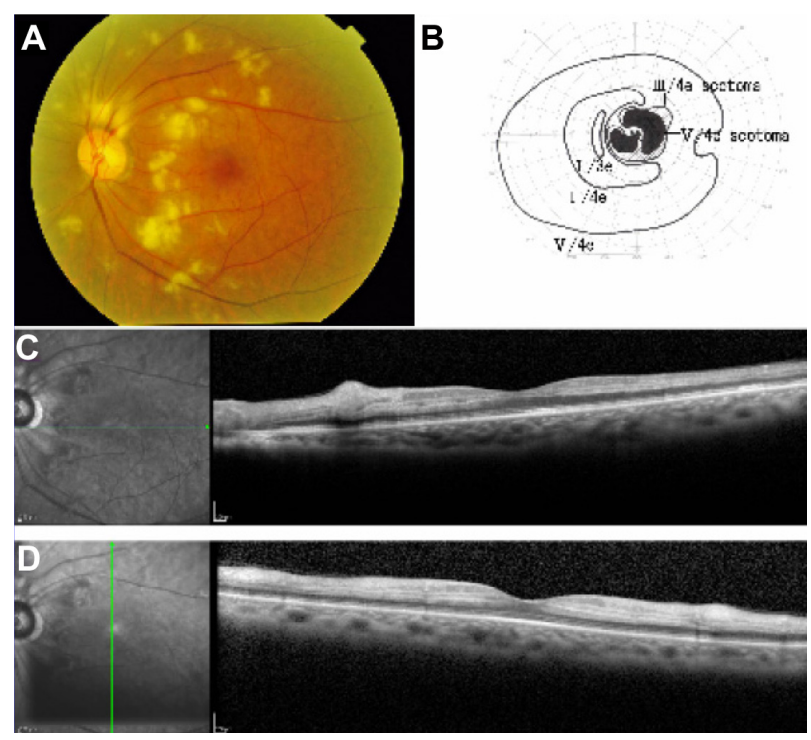

Figure I Clinical findings at presentation.

Notes: (A) A color photograph of the fundus of the left eye shows diffuse retinal whitening. (B) Goldmann kinetic perimetry of the left eye shows paracentral scotomas. (C and D) Optical coherence tomography of the left eye shows edema in the inner retinal layers.

nonperfused areas in the peripheral retina; however, there was no retinal neovascularization (Figure 2). A diagnosis of neovascular glaucoma of the left eye was made, and treatment with intravitreal bevacizumab injection $(1.25 \mathrm{mg} / 0.05 \mathrm{~mL})$ and a total of 2,764 shots of panretinal photocoagulation was initiated to rapidly decrease IOP and avoid peripheral anterior synechiae.

One month after treatment ( 3 months after the head injury), BCVA and IOP in the left eye recovered to 0.4 in decimal chart and $8 \mathrm{mmHg}$, respectively, under latanoprost treatment. Five months after the head injury, BCVA and IOP in the left eye were 0.4 in decimal chart and $7 \mathrm{mmHg}$, respectively, without hypotensive drugs. The angle neovascularization and white lesions gradually regressed (Figure $3 \mathrm{~A}$ ). The visual field defect partially recovered (Figure 3B). Optical coherence tomography showed retinal thinning in the inner layers (Figure 3C and D).

\section{Discussion}

To the best of our knowledge, this is the first reported case of neovascular glaucoma secondary to traumatic Purtscher's retinopathy. In a previous report, retinal neovascularization occurred following Purtscher's retinopathy, ${ }^{4}$ illustrating that retinal ischemia could be severe enough to induce neovascularization. In the present case, a delay in the arm-toretina circulation time and the presence of nonperfusion areas on fluorescein angiography could represent retinal hypoperfusion, which may have led to angle neovascularization. 


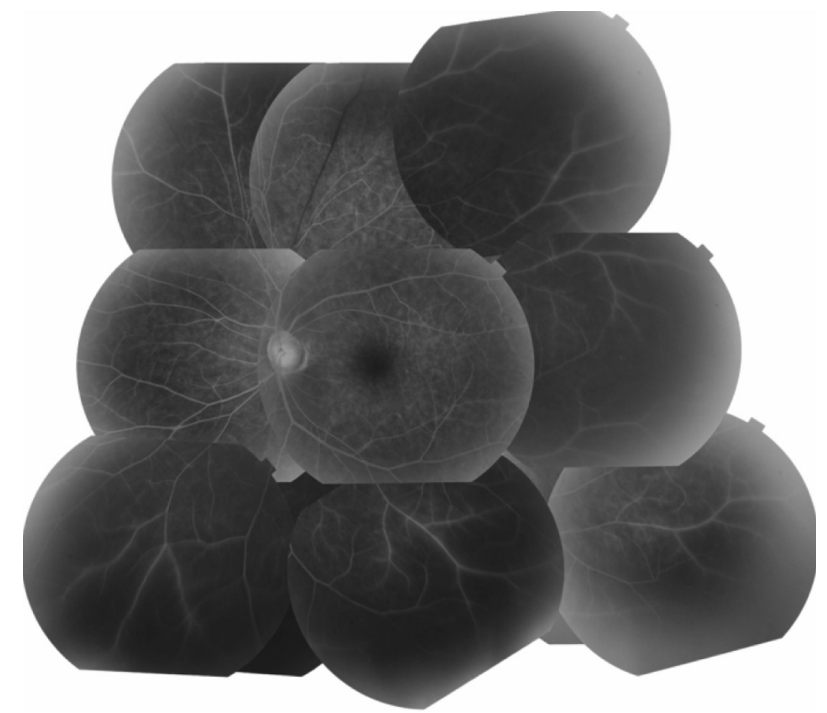

Figure 2 Clinical findings at I month after presentation ( 2 months after the head injury).

Notes: Fluorescein angiography of the left eye shows a mild leak from the peripheral vessels and from the nonperfusion areas in the peripheral retina; however, there is no retinal neovascularization. A delay in choroidal filling and arm-to-retina time was found.

The areas of nonperfusion on fluorescein angiography are much more extensive than their initial appearance on fundus examination; therefore, it might be helpful to consider earlier photocoagulation therapy if we performed fluorescein angiography at the initial visit.

We considered an alternative possibility that this case represented central retinal artery occlusion (CRAO) followed by neovascular glaucoma. Several cases of CRAO with cotton-wool-like patches on fundus examination and a good visual prognosis were reported. ${ }^{5}$ It is occasionally difficult to distinguish atypical CRAO from Purtscher's retinopathy; ${ }^{5}$ however, the patient's history of a head injury strongly suggests the diagnosis of Purtscher's retinopathy in the present case.

\section{Conclusion}

In conclusion, it is important to acknowledge that Purtscher's retinopathy can rarely lead to angle neovascularization and neovascular glaucoma. Careful follow-up is necessary to avoid long-term sequelae.

Clinical Ophthalmology

\section{Publish your work in this journal}

Clinical Ophthalmology is an international, peer-reviewed journal covering all subspecialties within ophthalmology. Key topics include: Optometry; Visual science; Pharmacology and drug therapy in eye diseases; Basic Sciences; Primary and Secondary eye care; Patien Safety and Quality of Care Improvements. This journal is indexed on

Submit your manuscript here: http://www.dovepress.com/clinical-ophthalmology-journal

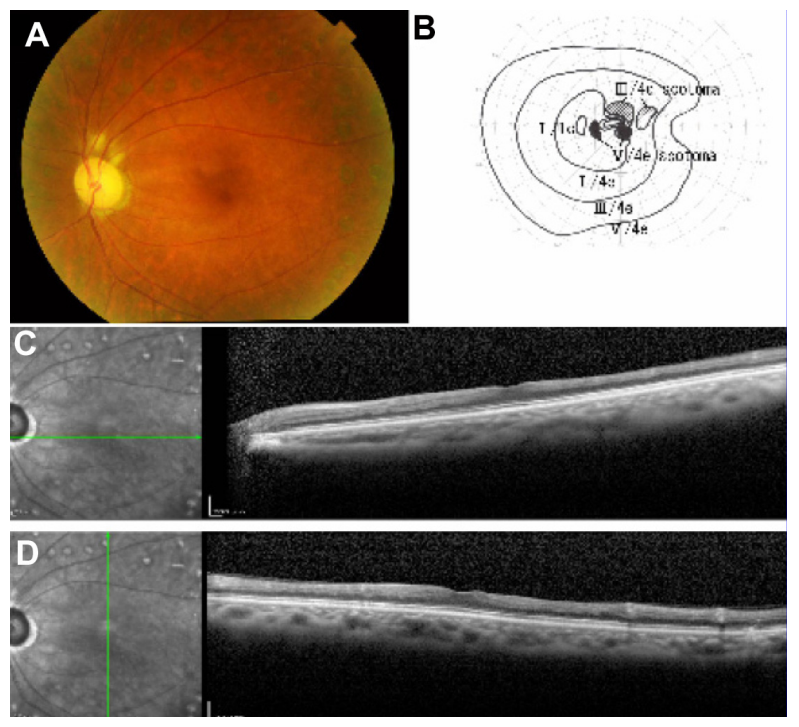

Figure 3 Clinical findings at 4 months after the initial examination ( 5 months after the head injury).

Notes: (A) A color photograph of the fundus of the left eye shows the regression of retinal white lesions. (B) Goldmann perimetry of the left eye shows the regression of the paracentral scotomas. (C and D) Optical coherence tomography of the left eye shows atrophy of the inner retinal layers.

\section{Disclosure}

The authors report no conflicts of interest in this work.

\section{References}

1. Purtscher O. Noch unbekannte befunde nach schadeltrauma. [Unknown findings after head trauma.] Ber Dtsch Ophthalmol Ges. 1910;36: 294-301. German.

2. Agrawal A, McKibbin MA. Purtscher's and Purtscher-like retinopathies: a review. Surv Ophthalmol. 2006;51(2):129-136.

3. Agrawal A, McKibbin M. Purtscher's retinopathy: epidemiology, clinical features and outcome. Br J Ophthalmol. 2007;91(11):1456-1459.

4. Chan A, Fredrick DR, Leng T. Neovascularization in Purtscher's retinopathy. Clin Ophthalmol. 2011;5:1585-1587.

5. Kurimoto T, Okamoto N, Oku H, et al. Central retinal artery occlusion resembling Purtscher-like retinopathy. Clin Ophthalmol. 2011;5: 1083-1088.
PubMed Central and CAS, and is the official journal of The Society of Clinical Ophthalmology (SCO). The manuscript management system is completely online and includes a very quick and fair peer-review system, which is all easy to use. Visit http://www.dovepress.com/ testimonials.php to read real quotes from published authors. 